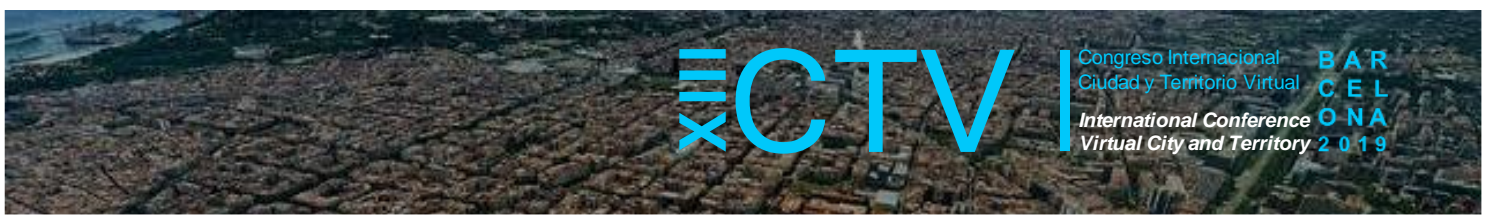

\title{
INTERVENCIONES URBANAS CON ENFOQUE DE SOSTENIBILIDAD: ESTRATEGIA CONTRA LA INFORMALIDAD EN MONTERÍA, COLOMBIA
}

\author{
Pinedo López, Jhon ${ }^{1 *}$; Lora Ochoa, Carmen ${ }^{2}$
}

Remisión inicial: 2019-06-27; Remisión definitiva: 2019-10-07; Publicación: 2019-12-21

Citación: Pinedo López, J. y Lora Ochoa, C. (2019). Intervenciones urbanas con enfoque de sostenibilidad: estrategia contra la informalidad en Montería, Colombia. En XIII CTV 2019 Proceedings: XIII International Conference on Virtual City and Territory: "Challenges and paradigms of the contemporary city": UPC, Barcelona, October 2-4, 2019. Barcelona: CPSV, 2019, p. 8702. E-ISSN 2604-6512. DOI http://dx.doi.org/10.5821/ctv.8702

\section{Resumen}

El objetivo central de esta investigación es analizar las intervenciones urbanas realizadas a partir de la aprobación del primer Plan de Ordenamiento Territorial de Montería (POT) en el año 2002 y sus efectos urbanos, principalmente las intervenciones diseñadas para corregir los desequilibrios urbanos asociados a la informalidad en el transporte (mototaxismo), comercio informal y uso inadecuado del espacio público. El enfoque del trabajo es cuantitativo y cualitativo; por un lado, se presentan datos de informalidad y de proyectos urbanos, y por el otro, se analizan sus efectos a nivel urbano y social. La metodología incluye técnica documental y técnica de campo. La primera corresponde a revisión del plan de ordenamiento territorial, planes de desarrollo municipal, informes institucionales y publicaciones. La segunda corresponde a la observación participante y a los registros fotográficos que permiten verificar la efectividad o la ineficacia de dichas intervenciones. Las fuentes de información a las que se recurre son, entre otras: POT de Montería 2002-2015 y los planes de desarrollo 2001-2003; 2004-2007; 2008-2011; 2012-2015 y 2016-2019.

Entre los resultados se encuentran: I) Indicadores sociales, urbanos y económicos de la ciudad de Montería antes del 2002, presentados en el diagnóstico del POT y descripción de las cinco principales estrategias de largo plazo de este plan, que permitieron establecer la hoja de ruta para la formulación de los futuros planes de desarrollo; II) La descripción de los principales proyectos e intervenciones urbanas programadas en los planes de desarrollo de los alcaldes Luis Jiménez Espitia, León Fidel Ojeda, Marcos Daniel Pineda García, en dos oportunidades, y Carlos Eduardo Correa. II) Programa "Ciudades Amables" como estrategia de movilidad eficiente y transporte público de pasajeros del Plan Nacional de Desarrollo 2006-2010 "Estado Comunitario: desarrollo para todos" aprobado mediante Ley 1151 de 2007 y la incorporación de Montería a este programa mediante documento CONPES No. 3638 del $1^{\circ}$ de febrero de 2010. IV) Plan Maestro de Cambio Climático PMCC y Plan de Acción 2032, Montería Sostenible, como soportes para lograr la inclusión de esta ciudad en el programa "Ciudades Sostenibles y Competitivas", que promueve la movilidad urbana sostenible; V) Proyectos "Sistema Estratégico de Transporte Público" (SETP), "Ronda del Sinú" y "Línea Azul", y sus efectos sobre la oferta de espacio público y la movilidad urbana.

Los proyectos programados en los planes de desarrollo posteriores al POT han contribuido a reducir parcialmente las actividades informales de comercio en andenes del centro, evidenciándose un significativo avance en materia de indicadores urbanos. La ejecución de éstos logró aumentar la oferta de espacio público de 1.66 metros cuadrados por habitante a 3.46, construir y reconstruir 133 parques, recuperar 144.604 metros cuadrados de andenes, construcción de 30 kilómetros de ciclovía y peatonalización de dos calles en el centro y norte de la ciudad. La movilidad para el peatón mejoró completamente en la avenida primera y de manera parcial en las avenidas segunda, tercera, cuarta y quinta, donde aún se mantienen algunos comerciantes antiguos y llegaron nuevos vendedores y/o mototaxistas.

Una de las conclusiones de este trabajo expresa que, los proyectos e intervenciones diseñadas con enfoque de sostenibilidad contribuyeron a mejorar la calidad ambiental y urbana de la ciudad y la calidad de vida de sus habitantes, pero no lograron cambiar los hábitos de los monterianos en el uso de transporte, principalmente aquellos relacionados con la obstinación por utilizar el transporte informal de la mototaxi, el uso innecesario de la motocicleta y la utilización reiterada del automóvil en carriles destinados para autobuses, lo cual evidentemente aumenta emisiones y no contribuye a la movilidad sostenible que se pretende con la visión de Ciudad Verde. Este artículo, resultado de

\footnotetext{
${ }^{1}$ Profesor e investigador Universidad Cooperativa de Colombia; http://orcid.org/0000-0001-9742-4296; ${ }^{2}$ investigadora Facultad de Derecho, Universidad Cooperativa de Colombia; https://orcid.org/0000-0002-1659-0952. ${ }^{*}$ Correo de contacto: ihon.pinedol@campusucc.edu.co
} 


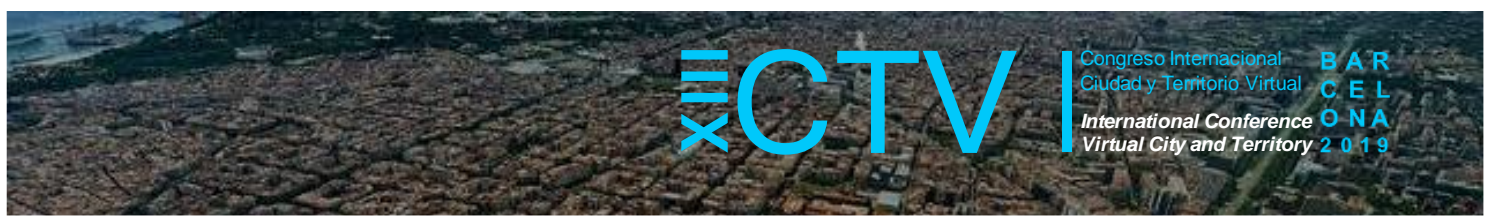

investigación financiada por la Universidad Cooperativa de Colombia, logra no sólo describir los proyectos urbanos de cinco períodos de la administración municipal, sino analizar su pertinencia, continuidad y efectividad a nivel urbano, social y de la movilidad. No hay evidencia en la actualidad de un trabajo similar en esta ciudad, aunque se encuentran informes, notas de prensa y trabajos científicos con aproximaciones parciales. Está dirigido a la comunidad académica y científica interesadas en este tipo de estudios.

\section{Abstract}

The main objective of this research is to analyze the urban interventions carried out after the approval of the first territorial planning plan of Montería (POT) in 2002 and its urban effects, mainly interventions designed to correct urban imbalances associated with informality in transport (motorcycle taxi), informal commerce and inappropriate use of public space. The work approach is quantitative and qualitative; on one hand, data on informality and urban projects are presented, and on the other hand, their effects are analyzed at urban and social levels. The methodology includes documentary technique and field technique. The first corresponds to a review of the territorial planning plan, municipal development plans, institutional reports and publications. The second corresponds to the participant observation and photographic records that allow verifying the effectiveness or ineffectiveness of the mentioned interventions. The sources of information used are, among others: POT of Montería 2002-2015 and the development plans 2001-2003; 2004-2007; 2008-2011; 2012-2015 and 2016-2019.

Among the results are: I) Social, urban and economic indicators of the city of Monteria before 2002, presented in the POT diagnosis and description of the five main long-term strategies of this plan, which allowed establishing the roadmap for the formulation of future development plans; II) The description of the main urban projects and interventions programmed in the development plans of the mayors Luis Jiménez Espitia, León Fidel Ojeda, Marcos Daniel Pineda García, twice, and Carlos Eduardo Correa. III) "Friendly Cities" Program as a strategy for efficient mobility and public transport of passengers of the National Development Plan 2006-2010 "Community State: development for all" approved by Law 1151 of 2007 and the incorporation of Montería to this program through CONPES document No. 3638 of February 1, 2010. IV) Climate Change Master Plan (PMCC) and the Action Plan 2032, Sustainable Monteria, as supports to achieve the inclusion of this city in the "Sustainable and Competitive Cities" program, which promotes the sustainable urban mobility; V) Projects "Strategic Public Transport System" (SETP), "Ronda del Sinú" and "Línea azul", and its effects on the offer of public space and urban mobility.

The projects programmed in the post-POT development plans have contributed to partially reduce informal trade activities on the center's platforms, showing significant progress in urban indicators. The execution of these managed to increase the supply of public space from 1.66 square meters per inhabitant to 3.46 , build and rebuild 133 parks, recover 144.604 square meters of platforms, construction of 30 kilometers of bicycle lanes and pedestrianization of two streets in the center and north of the city. The mobility for the pedestrian improved completely on the first avenue and partially on the second, third, fourth and fifth avenues, where some old merchants are still maintained and new vendors and / or motorcycle taxi drivers arrived.

One of the conclusions of this work expresses that, the projects and interventions designed with a sustainability approach contributed to improve the environmental and urban quality of the city and the quality of life of its inhabitants, but failed to change the habits of the monterians in the use of transport, mainly those related to the obstinacy to use the informal transport of the motorcycle taxi, the unnecessary use of the motorcycle and the repeated use of the car in lanes destined for buses, which obviously increases emissions and does not contribute to the sustainable mobility that It is intended with the vision of Green City. This article, as a result of research funded by Universidad Cooperativa de Colombia, not only describes the urban projects of five periods of the municipal administration, but also analyzes their relevance, continuity and effectiveness at the urban, social and mobility levels. There is currently no evidence of similar work in this City., although there are reports, press releases and scientific papers with partial approaches. It is aimed at the academic and scientific community interested in this type of studies.

Palabras Clave: proyectos urbanos; informalidad urbana; sistema estratégico de transporte público

Keywords: urban projects; urban informality; strategic public transport system

\section{Introducción}

Este trabajo presenta resultados del proyecto de investigación "Economía Informal en la ciudad de Montería; impacto sobre la ocupación, seguridad ciudadana e ingresos tributarios", financiado por la Dirección Nacional de Investigaciones de la Universidad Cooperativa de 


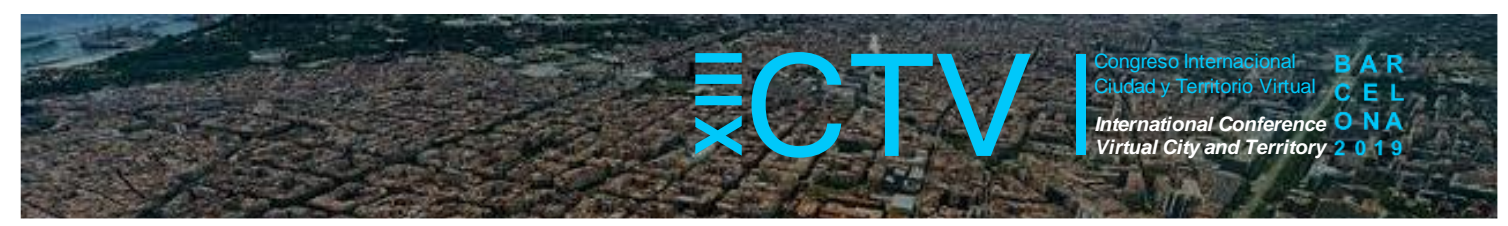

Colombia en el año 2017 y ejecutado a partir del 2018 por las facultades de Derecho y Ciencias Administrativas, Contables y Afines, sede Montería.

Inicialmente se describen algunos indicadores económicos, sociales y urbanos de la ciudad antes de la aprobación del POT y las principales intervenciones urbanas desarrolladas después de dicha aprobación en el año 2002. En esta descripción de indicadores se muestran problemas sociales, urbanos y ambientales, que no solo afectan la calidad de vida de los habitantes, sino que también generan tensiones sociales y conflictos entre deferentes grupos y/o actores de la sociedad.

Los indicadores, resultados y proyectos urbanos aquí presentados son obtenidos de los planes de desarrollo de las últimas seis administraciones, incluida la de Luis Jiménez Espitia, 20012003, administración en la que se formula y aprueba el POT y se establece la hoja de ruta seguida posteriormente por León Fidel Ojeda, 2004-2007, Marcos Daniel Pineda, 2008-2011, Carlos Eduardo Correa, 2012-2015 y nuevamente Marcos Daniel Pineda, 2016-2019.

Entre las intervenciones urbanas de mayor impacto sobresalen algunas realizadas con enfoque de sostenibilidad que buscan la recuperación del espacio público, ocupado por los comerciantes informales, el automóvil y la motocicleta. También, sobresalen proyectos relacionados con la construcción de equipamientos urbanos e infraestructura para mejorar la movilidad urbana, el transporte público colectivo y contrarrestar el fenómeno del mototaxismo.

La estructura de este trabajo académico es la siguiente: en primer lugar, se presenta la información geográfica y estadística de la ciudad; seguidamente, se describe la situación de Montería antes de la aprobación de su primer POT; en tercer lugar, se describen las intervenciones urbanas después del POT; posteriormente, se muestran las intervenciones con enfoque de sostenibilidad que pretenden corregir los problemas de espacio público, movilidad y transporte público colectivo en esta ciudad. Finalmente, se formulan las principales conclusiones del trabajo.

\section{Información geográfica y estadística de Montería}

Montería es una ciudad del caribe colombiano, fundada en 1777 y designada capital del departamento de Córdoba en 1952, después que éste fuera creado mediante Ley 9 de 1951 del 18 de noviembre y el Decreto Reglamentario 1392 de 1952 (Puche, 1988). Está ubicada a $750 \mathrm{Km}$ de Bogotá, $400 \mathrm{Km}$ de Medellín y a $300 \mathrm{Km}$ de Cartagena de Indias y su territorio municipal lo conforman importantes ecosistemas naturales como el río Sinú, la reserva natural Ciénaga de Betancí, el Cerro Sierra Chiquita y abundante vegetación, que enriquece el paisaje urbano, tal y como se puede apreciar en la figura 1.

Es a partir del año 1952 cuando comienzan a gestionarse importantes proyectos urbanos, que contribuirían posteriormente a convertirla en centro administrativo, económico y cultural de la región cordobesa y soporte de algunas subregiones de los departamentos de Antioquia y Sucre. Entre estos proyectos sobresalen el puente vehicular sobre el Río Sinú y edificios para la administración municipal y departamental. En el año 2002 es aprobado su primer Plan de Ordenamiento Territorial (en adelante POT). 


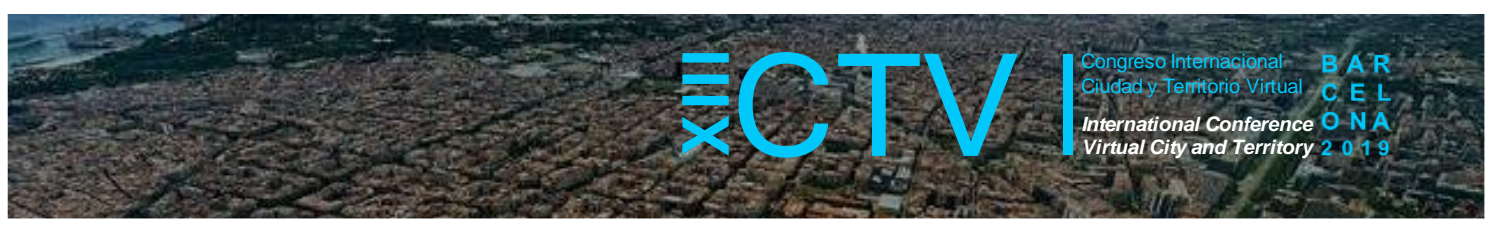

Figura 1. Paisaje urbano en el norte de Montería

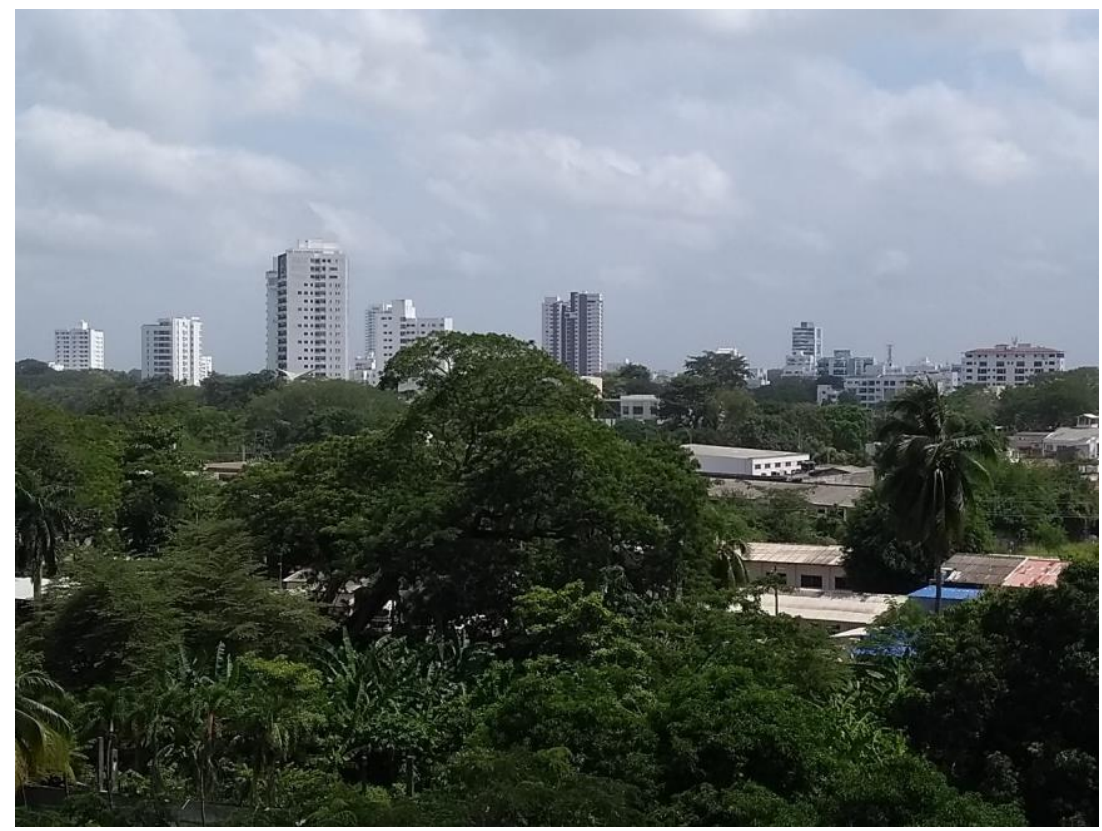

Fuente: Autores

El clima de Montería es cálido tropical, la temperatura promedio anual es de $28^{\circ} \mathrm{C}$, con picos que ascienden hasta los $40^{\circ} \mathrm{C}$ en horas del mediodía y tiene una humedad relativa promedio de $78 \%$. En cuanto a su demografía, la población proyectada para el municipio al año 2019 es de 362.757 habitantes en el área urbana y 103.242 en zona rural (DANE, 2019). El perímetro urbano asciende a 4.092 hectáreas, de un total municipal de 320.459 hectáreas (Alcaldía de Montería, 2016). Sus principales actividades económicas son ganadería, agroindustria y servicios. En la agroindustria se destacan empresas locales, regionales y nacionales dedicadas a la producción de lácteos, carnes y alimentos, mientras que en el sector de los servicios sobresalen empresas prestadoras de salud, centros comerciales, servicios financieros, clínicas de primer nivel y diez universidades privadas y una pública.

La Universidad de Córdoba es uno de los más grandes equipamientos de la ciudad con un área de 70 hectáreas y una comunidad académica que asciende a dieciocho mil integrantes (Universidad de Córdoba, 2017). Igualmente, sobresalen las universidades del Sinú, Pontificia Bolivariana, Cooperativa de Colombia, Corporación Unificada Nacional y Corporación Universitaria Remington.

Las intervenciones realizadas por la administración pública en las últimas dos décadas y las inversiones privadas le permiten a Montería contar actualmente con una gran oferta hotelera en el centro tradicional y en la zona norte, un aeropuerto de operación nacional ubicado por fuera del perímetro urbano, una terminal de transporte y una central de abastos en el sector nororiental y tres grandes superficies comerciales localizadas en el centro, norte y oriente de la ciudad. Asimismo, le permiten contar con significativos equipamientos, infraestructuras, proyectos multifamiliares de vivienda y megacolegios, que contribuyen a resolver de manera positiva los problemas sociales y urbanos originados en los años ochenta, como consecuencia del crecimiento demográfico, al cual aportó significativamente la llegada de desplazados del conflicto armado de la época. 


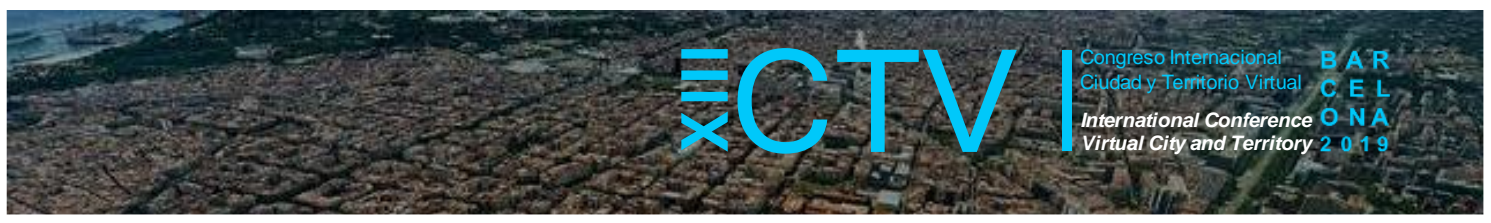

Es oportuno anotar que, hasta la década de los ochenta Montería fue una ciudad monocéntrica, en la que predominaba la arquitectura vernácula, la vivienda unifamiliar de planta baja, y en menor proporción, algunas expresiones de arquitectura republicana, neoclásica y moderna. Este período se caracteriza por un reducido parque automotor y el predominio de la bicicleta como medio de transporte. Sin embargo, después de la aprobación del POT la estructura urbana se modifica, el tráfico motorizado se intensifica, el servicio de transporte público masivo es insuficiente y surge el transporte informal de pasajeros en motocicleta, desencadenando graves problemas en la movilidad urbana.

\section{Montería antes del POT}

EI POT fue aprobado por el acuerdo 0018 del Concejo Municipal de esta ciudad en el año 2002, durante la administración del alcalde Luis Jiménez Espitia. En la fase de diagnóstico de este plan se encontraron, entre otros, los siguientes problemas: falta de identidad con el territorio; pérdida de la memoria histórica; déficit de espacio público; poco respeto por el espacio público; déficit de vivienda; marginalidad urbana; precaria infraestructura y deficiencia en el transporte público.

En el mencionado diagnóstico se identificaron treinta y cinco asentamientos informales con altos niveles de marginalidad e insostenibilidad, principalmente Los Bongos, El Cerro, Brisas del Sinú, Villa Jiménez e Invasión Sucre, que ocasionaban y siguen ocasionando, el mayor impacto negativo sobre el Río Sinú, humedales Villa Caribe y Villa Jiménez y la reserva natural de Sierra Chiquita. De igual forma, se identificaron problemas por inundaciones, alcantarillas a cielo abierto y afectación a la libre movilidad del peatón en la periferia y centro de la ciudad, tal y como lo muestra la figura 2.

Figura 2. Problemas urbanos en Montería
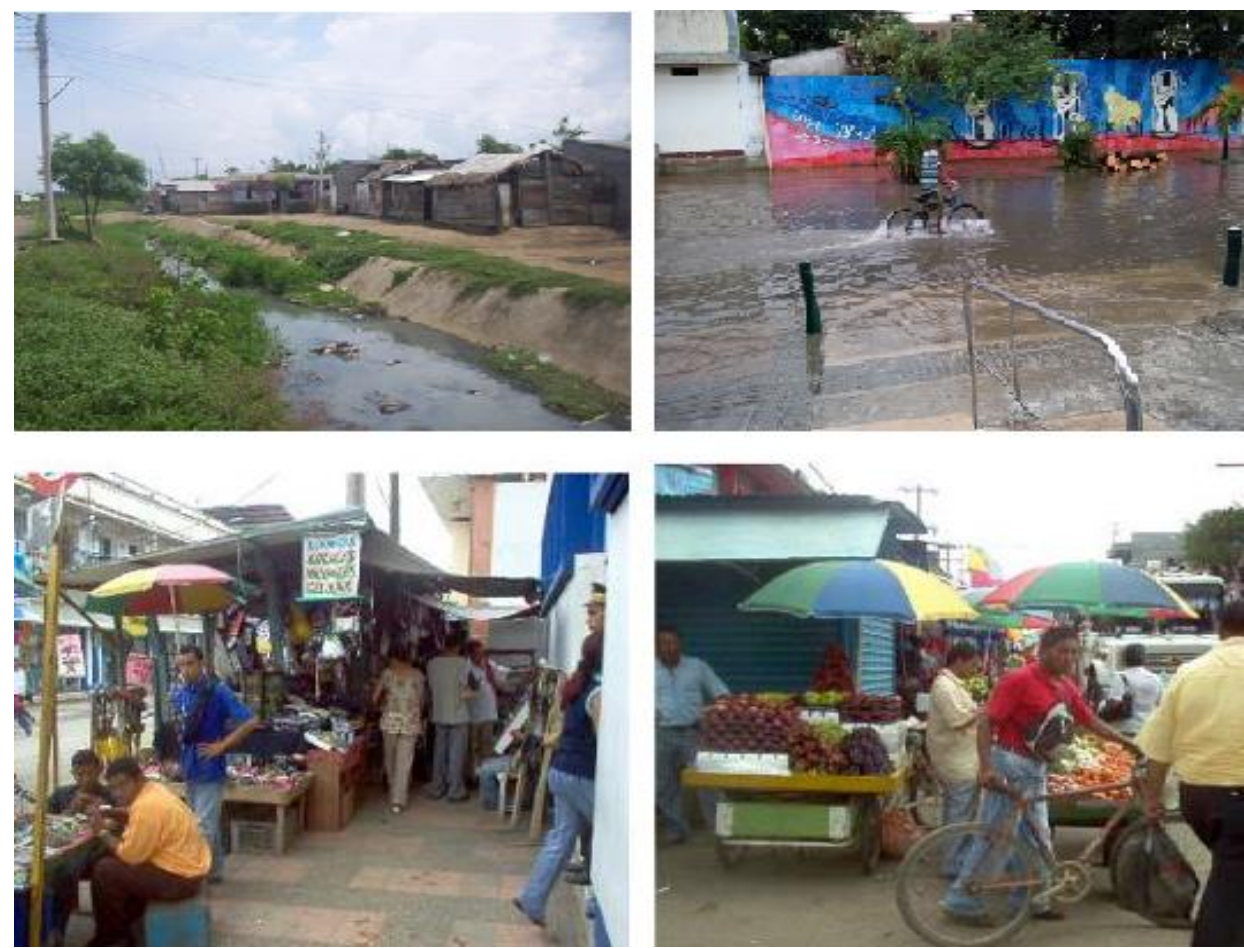

Fuente: Pinedo (2012) 


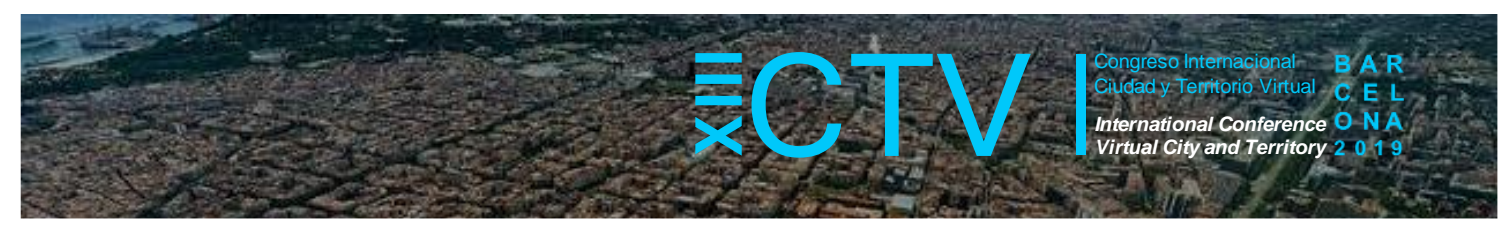

Estos niveles de informalidad en los asentamientos estaban acompañados de un déficit cualitativo de vivienda de 39.532 unidades, un déficit cuantitativo de 8.641 viviendas y baja oferta de espacio público, inferior a 1.60 metros cuadrado por habitante (Alcaldía de Montería, 2002). Se encontró una red viaria de 719.9 kilómetros con 231.9 Kilómetros pavimentados (32\%) y 10 kilómetros adoquinados (Pinedo, 2019). Indicadores similares se presentaban en la prestación de servicios públicos: el alcantarillado sanitario alcanzaba una cobertura del $25 \%$; el acueducto del $70 \%$; la recolección de residuos $80 \%$, el servicio de energía $90 \%$, desempleo de 13.9\% y una población urbana de 250.023 habitantes para el año 1998 (Negrete, 1999).

La principal fuente de empleo hasta los años ochenta fue la empresa pública, que incluía Alcaldía, Gobernación, Electrificadora de Córdoba, Universidad de Córdoba, Banco Ganadero, Caja Agraria, Bancos Popular, Central Hipotecario, Industrial y Comercial, Policía Nacional, Ejercito, Departamento Administrativo de Seguridad (DAS), Instituto de Mercadeo Agropecuario (IDEMA), Instituto Nacional de Adecuación de Tierra (INAT), Instituto Colombiano Agropecuario (ICA), Instituto Colombiano de la Reforma Agraria (INCORA), Empresa Nacional de Telecomunicaciones (TELECOM), Instituto de Bienestar Familiar, Hospital San Jerónimo y la Dirección de Impuestos y Aduanas Nacionales (Pinedo, 2012).

\section{Intervenciones urbanas a partir de la aprobación del POT}

Con este diagnóstico, el plan propone cuatro instrumentos de intervención integral para la ciudad: 1) operación de áreas marginales; 2) crecimiento programado; 3) configuración del centro urbano; 4) cualificación de áreas consolidadas. Además, establece acciones concretas para recuperación, mejoramiento y construcción de espacio público, vivienda, servicios públicos, equipamientos e infraestructuras viales y de transporte, que pretenden resolver necesidades básicas insatisfechas, informalidad urbanística e invasión del espacio público.

Las acciones descritas se materializaron mediante programas y proyectos en los planes de desarrollo de la presente y futuras administraciones municipales de Montería. En esta dirección, la hoja de ruta es fijada desde la administración municipal 2001-2003 de Luis Jiménez Espitia, quien incorpora cinco "megaproyectos", que se convertirían en punto de referencia para los siguientes alcaldes: 1) Sistema de Servicios Públicos Básicos; 2) Sistema Municipal Multimodal de Transportes; 3) Banco de Tierras; 4) Sistema Municipal de Aguas y Parques; 5) Montería, municipio cultural y educativo. Sin embargo, en este período surge y toma fuerza el mototaxismo como medio de transporte informal y se inicia un período de fuertes tensiones sociales que aún se mantienen (Restrepo y López, 2014).

En esta administración se realizaron los diseños de la terminal de transporte, la prolongación de algunas vías urbanas, un equipamiento comercial en el sur y el diseño de la primera fase del parque lineal de la Ronda del Sinú, proyectos incluidos en los megaproyectos "Sistema municipal multimodal de transportes" y "Sistema municipal de aguas y parques". Este último incorpora la recuperación de la avenida Primera y las reservas naturales Sierra Chiquita, Ciénaga de Betancí y Corredor del Río Sinú.

En la administración 2004-2007 de León Fidel Ojeda el plan de desarrollo "Construyamos el municipio por el camino correcto" incluyó el proyecto Ronda del Sinú y el programa de recuperación del espacio público "Rescatando Espacios para disfrutar la Construcción de la Ciudad por el Camino Correcto", que pretendía devolverles a los habitantes los espacios 


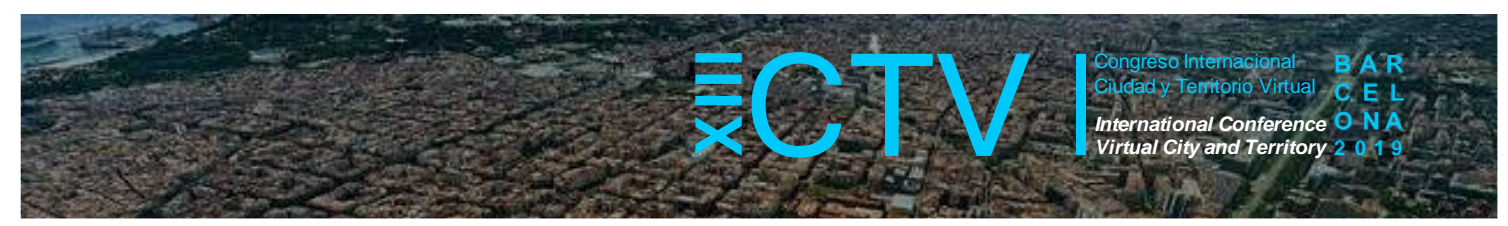

públicos usurpados por el comercio informal, los automóviles y la motocicleta. Asimismo, se desarrollaron obras de infraestructura para la ampliación de la cobertura de acueducto y alcantarillado, pavimento de vías y construcción de la terminal de transporte. No obstante, el espacio público recuperado fue poco, el mototaxismo siguió creciendo y la movilidad vehicular comenzó a presentar problemas.

El mototaxismo para este período se había convertido en un problema de dimensiones sociales, económicas y políticas, que reiteradamente alteraban el orden público. Ya en el año 2006 la prensa local informaba sobre la circulación de 3.000 mototaxistas y las afectaciones a los empresarios del transporte formal, quienes estimaban una reducción de 150.000 pasajeros por día, a expensas de este transporte informal (Restrepo y López, 2014). Esta situación llevó al alcalde León Fidel Ojeda a expedir el Decreto 0270 de 2006, que restringe el uso de la motocicleta en la zona urbana de Montería los martes de cada semana entre 6:00 AM y 8:00 PM; el uso de la moto con parrillero entre las calles 24 y 41 y carreras 1 y 13 de 6:00 AM a 8:00 PM de lunes a viernes y el uso de la motocicleta con parrillero los jueves de cada semana, entre las 6:00 AM y 8:00 PM. Estas medidas restrictivas continuarían en la siguiente administración, pero el problema de la informalidad en el transporte persistiría y al finalizar el período, el número de mototaxistas ascendía a 8.000 y los empresarios reportaban registro de 320 pasajeros diarios por autobús, cuando su proyección para el retorno de la inversión era de 500 pasajeros diarios (Castillo, 2010).

Para el año 2008 fue elegido Marcos Daniel Pineda, quien formuló el plan de desarrollo 20082011 "Juntos Hacemos Más", aprobado por Acuerdo municipal № 003 del 27 de mayo de ese mismo año por el Concejo de Montería. Este plan se estructura a partir de cinco grandes líneas estratégicas de acción, entre la cual se encuentra "Montería una ciudad amable y dinámica" que incluye proyectos de ampliación de la Red de alcantarillado, construcción de la Central de abastos, vías urbanas y mejoramiento del transporte público y la movilidad urbana.

En el plan se establecieron metas de recuperar 77.000 metros cuadrados de sectores deteriorados en el centro de la ciudad, remodelación de los dos parques centrales, jerarquización de los perfiles viales, peatonalización de calles, suprimir bahías de parqueo, ubicación de parqueos estratégicos, señalización de la ciudad, ampliación de andenes y recuperación del espacio público. Estas metas se consiguieron a través del proyecto "Plan Centro", que incluyó la intervención de las avenidas primera, segunda, tercera y cuarta, entre calles veintisiete y cuarenta y recuperación del espacio público en sectores parciales de las avenidas segunda y tercera.

También, se continuó con el proyecto Ronda del Sinú en su segunda fase, que incluía la zona norte, y que, junto a la zona central, demandaron una inversión cercana a los 4.5 millones de dólares (Alcaldía de Montería, 2011). El proyecto en mención se convirtió en emblema de la ciudad y con el propósito de garantizar su sostenibilidad y ampliación al sur y occidente se incluyó en el "Plan maestro de cambio climático: Montería ciudad verde", formulado y aprobado en el año 2011. En él se plantean soluciones a desarrollar en un horizonte de 10 años y se define que este parque es un proyecto de recuperación de la ribera con cinco intervenciones: centro, norte, sur, margen izquierda y sector Sucre, con un área total intervenida de 110.000 metros cuadrados, que comprende la siembra de 550 árboles. 


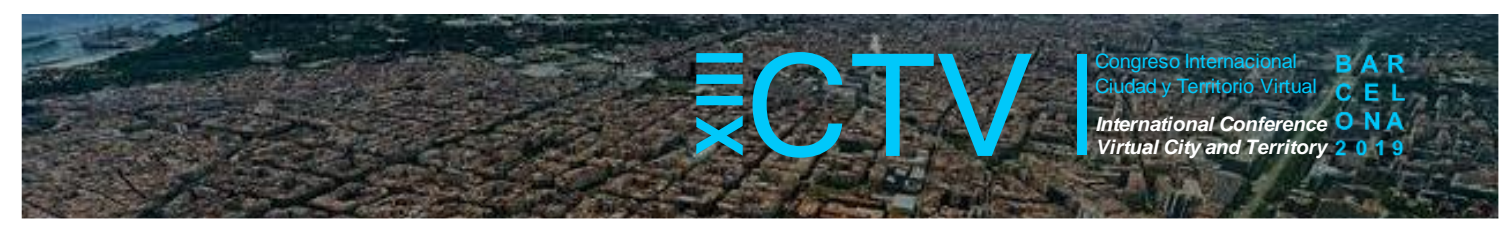

El Plan Maestro de Cambio Climático (PMCC) abarca entre sus ejes temáticos la movilidad urbana sostenible, que incluye dentro de sus proyectos el Sistema Estratégico de Transporte Público (SETP), Red de Ciclovías y Días verdes del transporte. Igualmente, incluye proyectos de saneamiento básico y agua potable, alcantarillado pluvial para la ciudad y el Plan Maestro de Parques, que establece una meta de construcción y rehabilitación de 96 parques, con un área de 320.000 metros cuadrados y la siembra de 2.000 árboles (Alcaldía de Montería, 2011).

El período 2008-2011 fue de gran aporte para la ciudad, por cuanto se logró la materialización de proyectos urbanos de infraestructura, equipamientos y espacio público. Sin embargo, las intervenciones urbanas de mayor impacto se lograrían en los períodos 2012-2015 en la administración de Carlos Eduardo Correa y 2016-2019, segunda administración de Marcos Daniel Pineda.

\section{Intervenciones urbanas con enfoque de sostenibilidad}

El Plan de Desarrollo 2012-2015 "Progreso para Todos", aprobado mediante Acuerdo 014 de 31 de mayo de 2012, se diseño con cinco ejes programáticos: 1) Montería, más social e incluyente; 2) Seguridad y Justicia para la Convivencia; 3) Infraestructura y Sostenibilidad para el Progreso; 4) Productividad y Competitividad, y 5) Gobernabilidad con pertenencia y corresponsabilidad. Esta administración continúa la dirección establecida por los planes anteriores, principalmente en materia de infraestructura y sostenibilidad, pero al igual que las administraciones antecesoras, se encontraba con el fenómeno creciente del transporte público informal en motocicletas.

Con el propósito de contener el transporte ilegal, el plan en su artículo 85 incorpora el subprograma "construcción del Sistema Estratégico de Transporte Público SETP, ciudades amables", dentro del eje "infraestructura y sostenibilidad para el progreso". El subprograma establece como metas la construcción de vías, senderos peatonales, ciclo rutas y espacios públicos del casco urbano para implementar el SETP. Además, incluye la construcción de la central de abastos, recuperación del centro de la ciudad en sectores aun no intervenidos por la administración, ampliación de las redes de alcantarillado en sectores marginados y cobertura del $100 \%$ en zona urbana y construcción de dos complejos deportivos.

La meta real establecida para el período en cuanto al SETP es la construcción de 33 kilómetros de vías, 248 paraderos, 123.000 metros cuadrados de andenes y espacio público, dos puentes vehiculares, cinco puentes peatonales. Otras metas son la intervención de 45.868 metros de canales; 60 parques, construcción de la sede de la alcaldía en la margen izquierda y la remodelación del mercado central (Alcaldía de Montería, 2012). Contribuye al logro de estos indicadores la inclusión de Montería en el programa Ciudades Amables. Es oportuno anotar que este programa hace parte de la estrategia de movilidad eficiente y transporte público de pasajeros del Plan Nacional de Desarrollo 2006-2010 "Estado Comunitario: desarrollo para todos" aprobado mediante Ley 1151 de 2007.

El programa "Ciudades Amables" con financiación del Banco Interamericano de Desarrollo y apoyo del gobierno central, se orientó a consolidar el sistema estratégico de transporte público (SETP), mediante: aumento de la infraestructura urbana; mejora de los carriles para transporte público; construcción de paraderos; estaciones y terminales de transferencia de pasajeros, y modernización de los sistemas semafóricos de control. Este proyecto demandó recursos por 


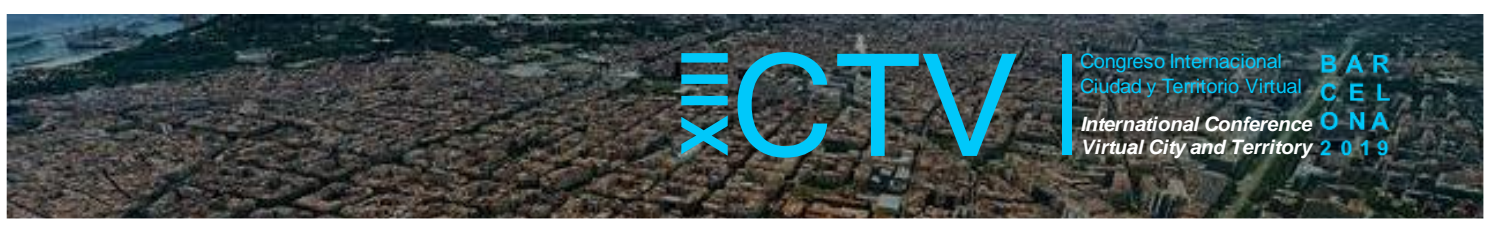

\$268.143.000.000 (aproximadamente 84 millones de dólares), para la reconstrucción de 33,17 kilómetros de vías, recuperación de 122.856 metros cuadrados de andenes y espacio públicos, $30 \mathrm{~km}$ de ciclovía, 5 puentes peatonales, 2 puentes vehiculares, 155 paraderos con cubiertas, 18 paraderos con espacio público, 6 centros descentralizados, 9 intercambiadores viales y una central semafórica (Montería Amable, 2019)

Ahora bien, los proyectos Ronda del Sinú y SETP, ambos con gran enfoque de sostenibilidad, quedarían, además, incluidos estratégicamente en el "Plan de Acción 2032, Montería Sostenible", aprobado en el año 2013. Esto contribuyó a que Montería fuera incluida posteriormente en el programa "Ciudades Sostenibles y Competitivas" que tiene por objetivo impulsar el desarrollo sostenible de las ciudades intermedias del país con una visión de largo plazo (FINDETER y BID, 2013). En este programa también se encuentran incluidas Pasto, Barranquilla, Bucaramanga, Manizales, Pereira, Cartagena, Valledupar, Armenia y Villavicencio. Con la visión de sostenibilidad, una hoja de ruta definida, resultados importantes en infraestructura y continuidad en los dos proyectos de largo plazo (Ronda del Sinú y SEPT) terminó el período 2012-2015 de Carlos Eduardo Correa e inició la segunda administración de Marcos Daniel Pineda, con su Plan de Desarrollo 2015-2019 Montería adelante, en el que se propuso proyectar a Montería como la Ciudad Verde de Colombia. Los ejes estratégicos de este plan son: 1) Montería ciudad verde y sostenible; 2) Ciudad inteligente; 3) Seguridad y convivencia para la paz; 4) Buen gobierno y participación ciudadana, y 5) Montería Inteligente.

El programa Montería Ciudad Verde incluyó los subprogramas "movilidad urbana sostenible" y "creación y recuperación de espacios públicos y zonas verdes", centrando gran esfuerzo por finalizar y consolidar el SETP y la Ronda del Sinú, considerada como proyecto de planificación estratégica (Babilonia, Sánchez, Jiménez Caldera y Durango, 2018). La Ronda del Sinú la conforman los parques Ronda Norte, con $1 \mathrm{~km}$; Ronda Centro, con 2,5 km, y Occidente, con $0.4 \mathrm{Km}$. La calidad de las zonas verdes de este parque en la zona centro se observa en la figura 3.

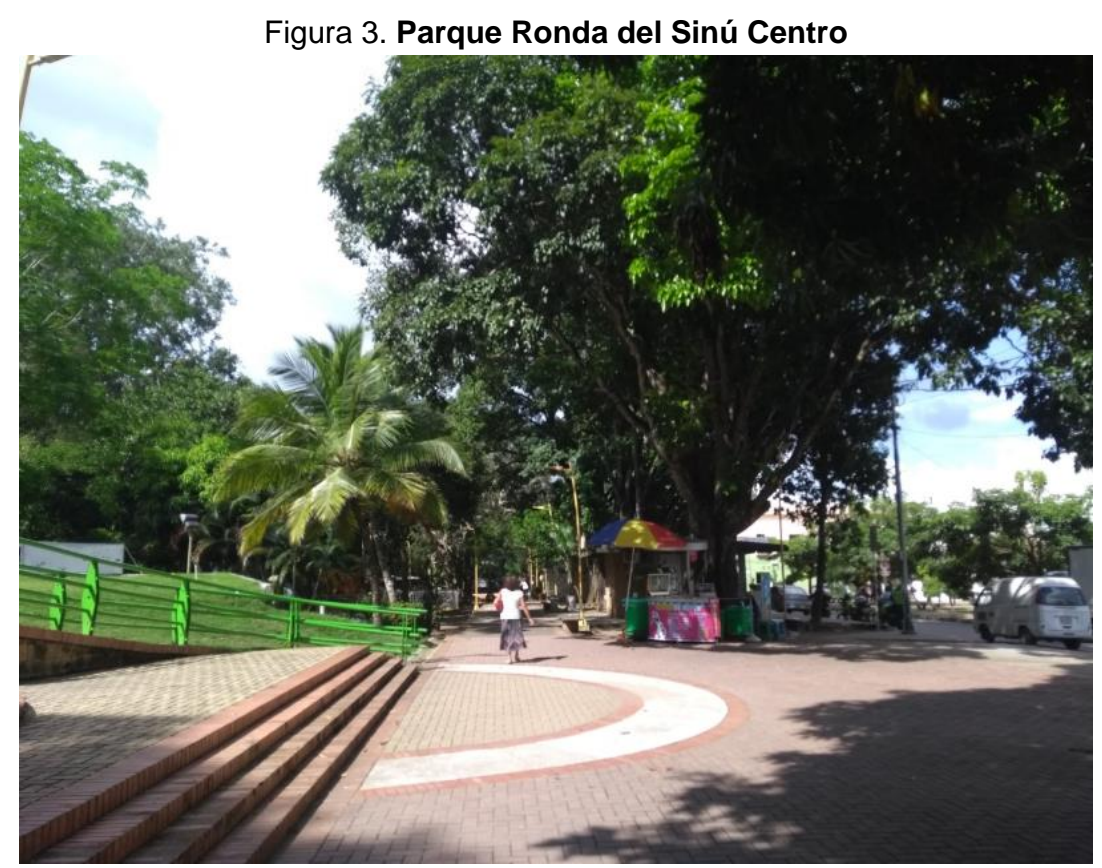

Fuente: Autores 


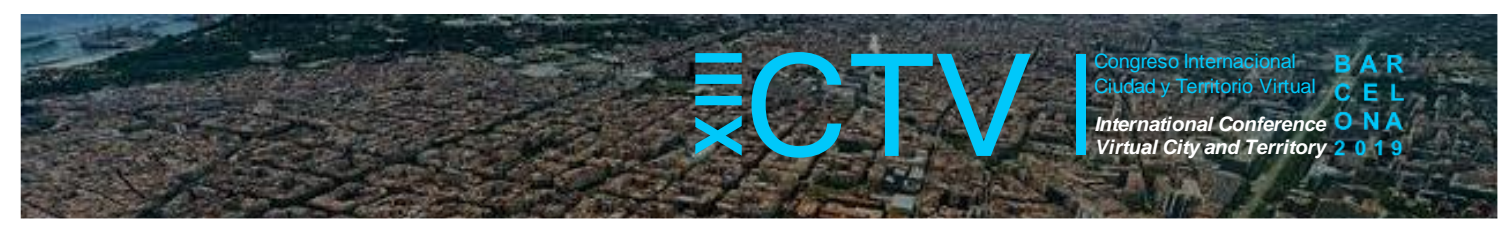

Otro importante proyecto relacionado con el SETP es el denominado "Línea Azul", que tiene como propósito mejorar la movilidad a través del río Sinú y convertirlo en articulador del territorio y eje turístico. Esta línea pretende ser integrada con el Sistema Estratégico de Transporte Público (SETP) e integrar el sur con el centro, el norte y el corregimiento de Los Garzones, proyectado para ser incorporado al perímetro urbano de Montería. Su primera fase está proyectada para una longitud de ruta de 3,8 kilómetros, con embarcaciones para 60 personas y una demanda estimada de 3.308 pasajeros por día, atendidos por una flota de cuatro naves iluminadas con energía solar (FINDETER y BID, 2018).

Los datos expuestos en este trabajo muestran un significativo avance de la ciudad en materia de indicadores urbanos, a partir de las intervenciones urbanas de las últimas seis administraciones. Éstas, lograron aumentar la oferta de espacio público de 1.66 metros cuadrados por habitante a 3.46, construir y reconstruir 133 parques, recuperar 144.604 metros cuadrados de andenes, la construcción de 30 kilómetros de ciclovía y la peatonalización de dos calles en el centro y norte de la ciudad (Rodríguez, 2016). De igual manera, lograron superar el déficit en la cobertura de servicios públicos domiciliarios y ampliarlos al $98 \%$ en agua potable, 99.5\% energía eléctrica, 92.5\% Gas domiciliario y 94.24\% alcantarillado al año 2017 (Superintendencia de Servicios Públicos, 2018).

Estos importantes logros permitieron reducir parcialmente las actividades informales de comercio en andenes del centro de la ciudad y en parques centrales y de la periferia, devolviendo andenes al peatón y áreas libres a los ciudadanos, que por varias décadas fueron invadidas por vendedores informales estacionarios. La movilidad para el peatón mejoró completamente en la avenida primera y parcialmente en las avenidas segunda, tercera, cuarta y quinta, donde aún se mantienen algunos comerciantes antiguos y llegaron nuevos vendedores $\mathrm{y} / \mathrm{o}$ mototaxistas.

No obstante, en materia de transporte y movilidad sostenible, el SETP no ha sido la solución hasta el momento, primero, porque aún falta la incorporación de los buses articulados que deben reemplazar a los actuales, y segundo, porque las estrategias utilizadas para concientizar a la población sobre la importancia del transporte público y/o de la utilización de medios no motorizados a la fecha no surten efecto. Con respecto al sistema, es pertinente destacar que se construyó la infraestructura requerida, se diseñaron las rutas necesarias y el municipio y las empresas de transporte realizaron las inversiones pertinentes, pero la respuesta de los monterianos no retribuye aún los esfuerzos realizados.

La no retribución se evidencia en la congestión generada por el uso excesivo del automóvil y la motocicleta y en el bajo porcentaje de la población que utiliza el transporte público colectivo 0 modos no motorizados. Solo el $22 \%$ se transporta en autobús y el $9 \%$ en bicicleta (Zea y Palau, 2018). Sin duda alguna, estos bajos porcentajes obedecen al aumento del número de motocicletas y de personas dedicadas a la actividad del mototaxismo, tal y como lo muestran las cifras de diferentes estudios (Castillo, 2010; Restrepo y López, 2014; Gobernación de Córdoba, 2017). Evidencia de la actividad del mototaxismo se muestra en la figura 4.

Con relación al aumento de motocicletas, la ciudad pasó de tener 5.000 de estos vehículos en el año 2000 a 41.136 en el 2016, es decir, 9.232 motocicletas por cada 100.000 habitantes (Gobernación de Córdoba, 2017). Por su parte, la oferta de transporte público de pasajeros la conforman 285 autobuses (Burgos, y Lozano, 2016). Según la actual administración, en un día 


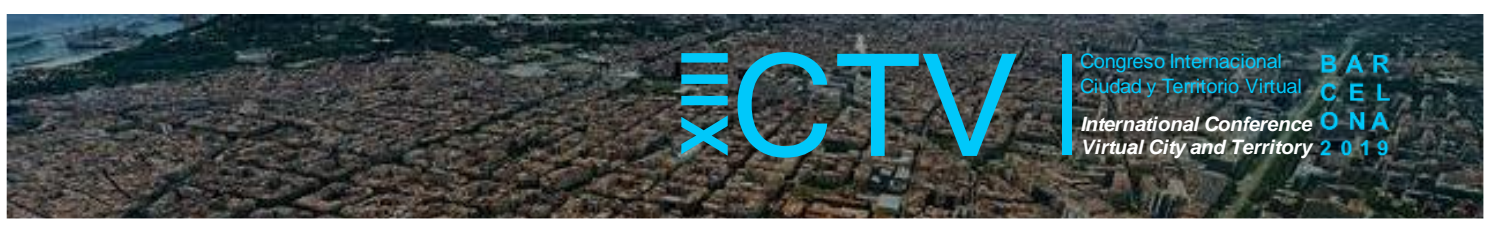

sin restricción de motos se transportan en autobús 81.000 personas, en mototaxi 46.000 , en Taxi 15.000 y en moto propia 22.000 (Alcaldía de Montería, 2016).

Figura 4. Preferencia por el transporte en mototaxi

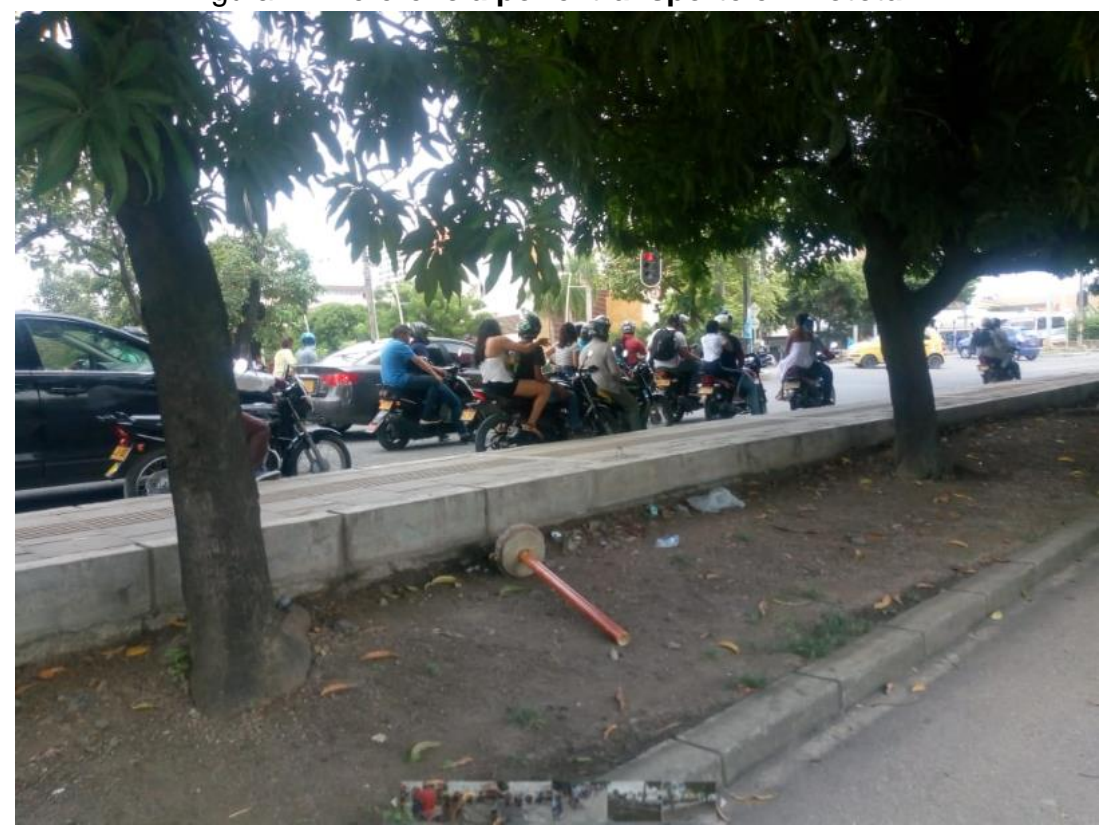

Fuente: Autores

A las cifras mencionadas se suman los accidentes y las transgresiones permanentes a las normas establecidas por la autoridad municipal para frenar el transporte informal. Al respecto, la Gobernación de Córdoba (2017) presenta las siguientes estadísticas: para la zona urbana de Montería: año 2015, 601 accidentes, 94 muertos, 1.135 lesionados; año 2016, 608 accidentes, 113 muertos y 1.005 lesionados y 135 accidentes por imprudencias de los motociclistas en los dos años. Igualmente, reportan 27.759 sanciones por infracciones de tránsito en los dos años, con 3.600 sanciones por conducir sin licencia, 2500 sin uso del casco y 618 por actividades de mototaxismo en el 2016.

Finalmente, las evidencias y estadísticas aquí expuestas permiten corroborar que el mototaxismo y el alto número de motocicletas en esta ciudad no contribuye a lograr la movilidad sostenible, ni la ciudad verde que se propusieron las últimas administraciones de Montería. No obstante, esta situación no resta mérito a los planes de desarrollo formulados por los mencionados alcaldes e invita a la reflexión de cada uno de los actores de la sociedad, quienes son los responsables de utilizar los medios de transportes mas amigables con el medio ambiente.

\section{Conclusiones}

El primer POT de Montería, aprobado en la administración de Luis Jiménez Espitia, 2001-2003, se constituyó en la herramienta efectiva para corregir gran parte de los problemas urbanísticos, sociales y económicos que arrastraba la ciudad desde antes de su constitución como capital de Departamento en el año 1952, estableciendo, además, la hoja de ruta adecuada para las 


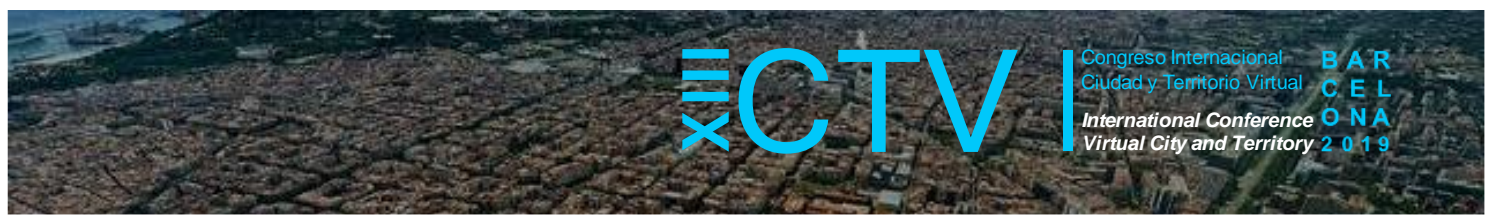

administraciones municipales de León Fidel Ojeda, Marcos Daniel Pineda y Carlos Eduardo Correa.

Los cuatro planes de desarrollo posteriores siguieron la ruta de sostenibilidad formulada por el POT, programando los proyectos e intervenciones pertinentes para resolver el déficit de vivienda, infraestructura, espacio público, equipamientos y los problemas asociados al transporte público de pasajeros y movilidad urbana, con una visión de ciudad sostenible.

Los proyectos e intervenciones diseñadas con enfoque de sostenibilidad contribuyeron a mejorar la calidad ambiental y urbana de la ciudad y la calidad de vida de sus habitantes, pero no lograron cambiar sus hábitos en el uso de transporte, principalmente en la utilización del transporte informal, la motocicleta y el automóvil, lo cual evidentemente aumenta emisiones y no contribuye a la movilidad sostenible que se pretende.

Las autoridades han intentado frenar el uso de la motocicleta como transporte informal mediante campañas de sensibilización y restricciones al uso de este vehículo desde el año 2006 con el Decreto 2961 en la presidencia de Álvaro Uribe Vélez y con el Decreto 0270 del mismo año en la Alcaldía de León Fidel Ojeda, restringiendo su uso un día a la semana (martes) y prohibiendo el parrillero otro día (jueves), así como la circulación con parrillero entre las calles 24 y 41 y entre las carreras 1 y 13, de lunes a viernes, de 6:00 AM a 8:00 PM. Sin embargo, se evidencia un abierto desafío de los mototaxistas a las autoridades, la obstinación de la población que utiliza este transporte ilegal y la falta de autoridad para sancionar a los trasgresores.

Las restricciones establecidas se mantienen vigentes, con la novedad del levantamiento de la restricción del uso de la moto el martes de cada semana, a través del Decreto 0002 del 4 de enero de 2016 y ratificado por el Decreto 0002 del 4 de enero de 2019, en el que se establece la utilización de la motocicleta ese día, sin parrillero. Esta decisión favoreció a quienes la utilizan como su medio de transporte particular, pero estimuló más la actividad del mototaxismo, tal y como se evidencia en las calles todos los días y como lo revelan las estadísticas suministradas por la Gobernación de Córdoba (2017), relacionadas con la actividad del mototaxismo.

Consideramos que, la complejidad del fenómeno del mototaxismo y los efectos que genera en la movilidad urbana, seguridad ciudadana y en las relaciones sociales y económicas, lo constituyen en objeto de investigación de gran interés, no solo en el área del urbanismo, sino en otras disciplinas como la economía, la sociología y el derecho.

Agradecimientos: a la Universidad Cooperativa de Colombia por su financiación al proyecto "Economía Informal en la ciudad de Montería, Colombia: impacto sobre la ocupación, la seguridad ciudadana e ingresos tributarios" y su apoyo para la participación en el XIII Congreso Internacional Ciudad y Territorio Virtual CVT

Contribuciones de los autores: los autores participaron conjuntamente en cada uno de los apartados.

Conflicto de Intereses: Los autores declaran que no hay conflicto de intereses 


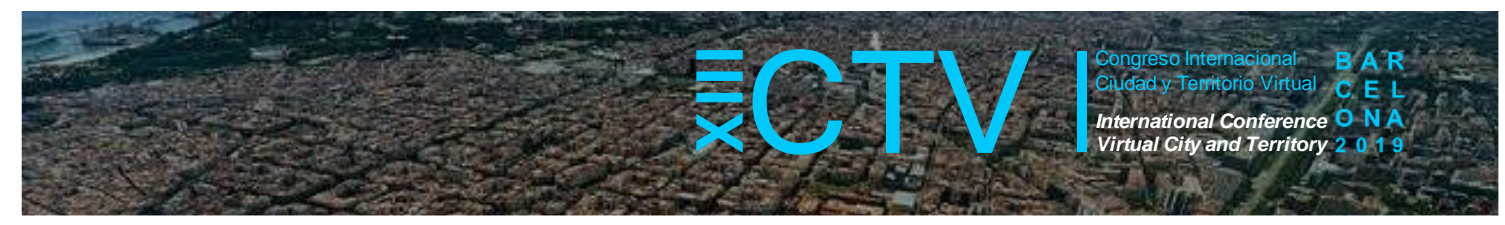

\section{Bibliografía}

Alcaldía de Montería (2002). Plan de Ordenamiento Territorial 2002-2011. Montería, Colombia. Autor.

Alcaldía de Montería (2011). Plan Maestro de Cambio Climático: Montería Ciudad Verde 2019. Recuperado de http://www.minambiente.gov.co/images/AsuntosMarinosCosterosy RecursosAc uatico/PICC CIUDAD VERDE MONTERIA.pdf

Alcaldía de Montería (2012). Plan de Desarrollo 2012-2015 Progreso para todos. Recuperado de https://www.monteria.gov.co/documentos/156/plan-de-desarrollo-alcaldia-de-monteria/

Alcaldía de Montería (2016). Plan de desarrollo Montería Adelante 2016-2019. Recuperado de http://cvsaulaverde.com/administracion/documentos/201609260.56117000\%201474894815PLA N-DESARROLLO-MONTERIA-2016-2019.pdf

Babilonia, R.; Sánchez, R.; Jiménez Caldera, J. y Durango, G. (2018). Planificación urbana estratégica a través de macroproyectos urbanos: percepción ciudadana sobre el parque lineal "La Ronda del Sinú" en la ciudad de Montería, Colombia. Revista de Urbanismo, (39), 1-12.

Burgos, D. y Lozano, L. (2016). Evaluación de la calidad del servicio de transporte público en Montería. (Tesis de pregrado). Universidad Cooperativa de Colombia, Montería.

Castillo, B. (2010). El mototaxismo ¿problema informal o un medio de generación de ingresos para resolver en parte el problema del desempleo en Montería? Económicas CUC, 31 (1), 89 104.

DANE (2005). Estimaciones y proyecciones de población. Recuperado de https://www.dane.gov.co/index.php/estadisticas-por-tema/demografia-ypoblacion/proyeccionesde-poblacion

FINDETER y BID (2013). Plan de Acción 2032. Montería Sostenible. Recuperado de https://issuu.com/findetersa/docs/monteria baja

FINDETER (2018). Proyecto de sistema de transporte fluvial de pasajeros del río Sinú: una realidad. Recuperado de https://www.findeter.gov.co/publicaciones/403257/proyecto de sis tema de transp orte fluvial de pasajeros del rio sinu una realidad

Montería Amable (2019). Sistema Estratégico de Transporte Público. Recuperado de http://www.monteriaamable.gov.co/index.php?lang=es

Negrete, V. (1999) Los asentamientos subnormales de Montería. Montería, Colombia: ACNURUniversidad del Sinú.

Pinedo, J. W. (2012). Urbanización marginal e impacto ambiental en Montería. Tesis doctoral, U.P.V., Valencia, España. Recuperado de http://riunet.upv.es/handle/10251/16468\#>. 2012

Puche, E. (1988): Crónicas y Estampas Monterianas. Montería, Colombia: Gráficas Urra. 


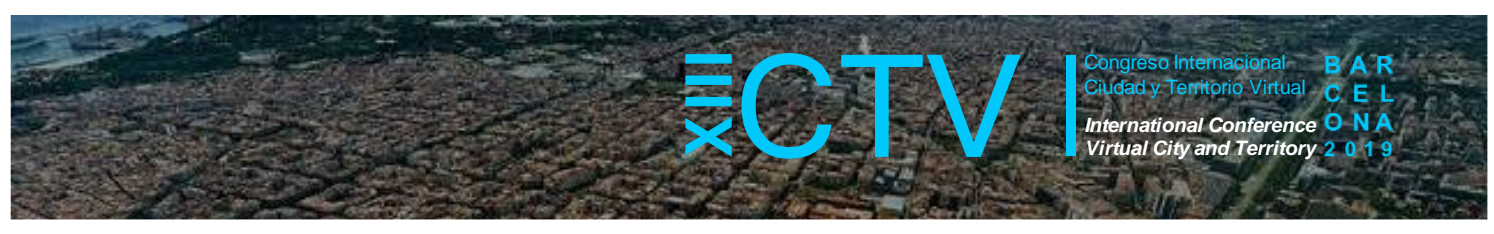

Restrepo, M. y López, L. (2014). El mototaxismo en relación con la imagen de ciudad y del ciudadano: Una mirada desde la prensa local en la ciudad de Montería. Miradas (12), 37 - 51.

Rodríguez, J. (2016). Montería Ciudad Verde. Recuperado de https://es.slideshare.net/jotapal1637/monteria-ciudad-verde-

Superintendencia de Servicios Públicos (2018). Evaluación integral de prestadores: Proactiva Aguas de Montería S.A. E.S.P. INF. 003. Bogotá, Colombia.

Universidad de Córdoba (2017). Boletín Estadístico. Recuperado de http://unicordoba.edu.co/files/BOLETN-ESTADSTICO-05-2017.pdf

Zea, R. y Palau, A. (2018). El Transmilenio del Río Sinú. Revista Semana, sección medioambiente, septiembre 05. Recuperado de https://www.semana.com/contenidoseditoriales/monteria-diez-anos-despues/articulo/el-transmilenio-del-rio-sinu/566466 